\title{
Performance of Zero forcing Pre-coder for Vectored DSL
}

\author{
Rajani Katiyar ${ }^{1}$ Padmaja K.V. ${ }^{2}$ \\ ${ }^{I}$ Electronics and Communication, RVCE Bangalore, India \\ ${ }^{2}$ Electronics and Instrumentation, RVCE Bangalore, India
}

\begin{abstract}
This paper deal with the performance analysis of vectored DSL in terms of the computational complexity and practical implementation. Paper is more specific for the design of pre-coder in downstream transmission of vectored DSL. There are many type of linear and nonlinear pre-coders have been proposed in the past. All of these pre-coder are either highly complex in design or they give poor performance in terms of bit rate. As the cost of the cp modem is an important issue, any technique that can minimize the complexity of the system is extremely beneficial. In this paper performance of basic model of vectored DSL is been analyzed using DSK 6713 a digital signal 32 bit, floating point processor. Here the clock frequency of processor is considered as $250 \mathrm{MHZ}$. This processor has advanced very long instruction word (VLIW) architecture, with performance of up-to 2400 (532) million instructions per second. The execution time of the processor is compared with the Matlab profiler function.
\end{abstract}

Keywords: Signal Processor Starter Kit (DSK), Digital Subscriber line (DSL), Very-high-bit-rate digital subscriber line (VDSL),Discrete multi tone modulation (DMT), power spectral density (PSD).

\section{Introduction}

In Broad-band Communication, Digital subscriber Line (DSL) technology provides High Speed Communication over twisted pair wire. The interference from one wire to other at near and far end create severe distortion on processing of data. Most of the research work is concerned with the minimization of this cross talk, so that more and more number of data bit per second can be transmitted over channel. To minimize the effect of cross talk, spectrum management technique such as static spectrum management and dynamic spectrum management came into existence.[1] Dynamic spectrum management (DSM) is better as compared to Static spectrum management because of many adaptive bit loading algorithms present in Dynamic Spectrum Management. Dynamic spectrum management is further classified into three forms viz., Level- 1, Level-2, and Level-3 also known as vectored DSL.

Vectoring is also known as cross talk cancellation. The purpose of using vectoring is to increase the data rate up to $52 \mathrm{Mbps}$ at frequency range of $30 \mathrm{MHZ}$. vectoring can increase the data rate of VDSL2 and G. Fast systems which is highly affected by far-end crosstalk. Vectored DSL transmission is working on the principle of MIMO mostly use for wireless transmission [2]. MIMO DSL is different from wireless MIMO system. The number of transmitting and receiving antennas are same in MIMO DSL but reverse in case of wireless MIMO communication. Using MIMO DSL, signals are jointly process in all the wires present in a binder. In downstream vectored transmission, the modems are present at the central office (CO). Pre-coder is used after modem to pre-distort the signal prior to transmission. The amount of pre- distortion is depending on crosstalk introduced in a binder. This technique is used to provider crosstalk free transmission, and increase the bit rate. Different type of pre-coder [3] [4] is use for downstream vectored transmission. Each pre-coder work on Different algorithms. Zero forcing and Tomlinson-Harashima pre-coder pre-coder very famous among them design of Tomlinson-Harashima pre-coder is very complex due to nonlinear architecture [5] [6]. Design of Zero forcing pre-coder is much simple then others.

In this paper, performance of pre-coder for downstream vectored DSL(VDSL-2) are analyzed with and without precoding. We Perform the bit rate analysis for the basic model in Matlab and on DSK 6713 Digital signal processor. We have shown that how the computational complexity [7][8] and execution time can be minimized in presence of DSK processor as target. Channel modeling is done for the scenario when all the users are saturated at different length.The paper is arranged as follows. Section II explains about basic system model. Section III gives detail about precoding tech for downstream vectored transmission. Simulation result comparing the bit-rate performance with and without precoding is presented in section IV. Our conclusion and future scope are summarized in section V.

\section{System Model}

Vectored DSL network consist of set of users $\mathrm{N}=\{1, \ldots, \mathrm{N}\}$ (modems) which operate on different frequency tone K known as Sub-carriers. Transmission of data is perform by DMT modulation technique. DMT modulation [9], maintain the orthogonality between each user. The system output is given by equation (1) 
$\mathrm{Y}_{\mathrm{k}}=\mathrm{H}_{\mathrm{k}} \mathrm{X}_{\mathrm{k}}+\mathrm{N}_{\mathrm{k}}$

The vector $X_{k}=\left\{x_{k}^{1}-\cdots-x_{k}^{n}\right\}$ represent the transmitted signal of all user on different frequency tone $\mathrm{k}$, where $\mathrm{x}_{\mathrm{k}}^{\mathrm{n}}$ represent the transmitted signal by user $\mathrm{n}$ on frequency tone $\mathrm{k} \cdot \mathrm{y}_{\mathrm{k}}^{\mathrm{n}}$ is the received signal of user $\mathrm{n}$ on frequency tone $\mathrm{k}$. Hk is the $\mathrm{N}^{*} \mathrm{~N}$ matrix. The transmit PSD of user $n$ on frequenctone $k$ is defined by $\mathrm{s}_{\mathrm{k}}^{\mathrm{n}}$. The bit rate of the $\mathrm{n}^{\text {th }}$ user on frequency tone $\mathrm{k}$ (here $\mathrm{n}$ and $\mathrm{k}$ both are variables) is given by

equation(2)

$$
b_{k}^{n} \triangleq \log _{2}\left(1+\frac{1}{\Gamma} \frac{\left|h_{k}^{n, n}\right|^{2} s_{k}^{n}}{\sum_{m \neq n}\left|h_{k}^{n, m}\right|^{2} s_{k}^{m}+\sigma_{k}^{n}}\right),
$$

Total data rate of the $\mathrm{n}^{\text {th }}$ user is given by equation (3) where $f_{s}$ is the DMT symbol rate, have value of 4000 .

$R^{n}=f_{s} \sum_{k} b_{k}^{n}$,

\section{Vectored Transmission}

Vectored transmission for DSL is classified in three main scenarios. Downstream vectored transmission, upstream vectored transmission and joint vectored transmission in both the direction. Precoding is used in downstream direction of vectored transmission [2]. Cancellation at the receiver is done in upstream direction of vectored transmission. In joint transmission precoding and cancellation is performed in downstream and upstream direction respectively. Downstream Transmission: For vectored transmission in downstream direction, the interference cancellation is impossible on the receiver side. But cross talk cancellation can be done on transmitter side as the signals are transmitted from the same place. Precoding is a technique that is use for cross talk cancellation. It pre-distort the signal before transmission so that the signal arrive at $\mathrm{CP}$ (customer premises) are crosstalk free. There are two algorithms that are used for precoding in downstream vectored DSL. Zero Forcing (ZF) pre-coder [10], and the Tomlinson-Harashima Pre-coder (THP) [11].

Zero-Forcing: It is type of linear pre-coder which use the inverse of channel for precoding. The input signal is predistored with the inverse of the channel before transmission. Pre-distortion increase the power of the transmit signal above the spectral mask constraint. Ref [12] showed that if all the transmitter is co located the channel matrix is RWDD which gives the optimal result. In RWDD channel matrix [12] [13] the diagonal and off-diagonal signal travels same distance but the off-diagonal signals a result of electromagnetic coupling between twisted pair wire. In RWDD matrix the diagonal element, $\left[\mathbf{H}_{k}\right]^{(n, n)}$, dominates the off diagonal element on the same row $\left[\mathbf{H}_{k}\right]^{(n, m)}$,

$$
\left|h_{k}^{n, m}\right| \leq \alpha_{k}\left|h_{k}^{n, n}\right|, \forall m \neq n .
$$

Where $\alpha_{\mathrm{k}}$ is define as

$$
\alpha_{k} \leq K_{\mathrm{xf}} \cdot f_{k} \cdot \sqrt{d_{\text {coupling }}},
$$

here $K \mathrm{xf}=-22.5 \mathrm{~dB}$ and $f k$ is the frequency on particular tone $k$ in MHz [3].If $\alpha_{\mathrm{k}}$ is not dependent on any binder configuration then $d_{\text {coupling }}$ that is coupling length between two wire constant $1.2 \mathrm{~km}$, that maximum length for VDSL3[14][15].The scaling factor, $\beta_{k}$ diag is used on the precoding matrix to insure the PSD mask constraint.

$$
\begin{aligned}
& \beta_{k}^{\text {diag }}=\max _{n} \sum_{m \in \mathcal{N}}\left|\left[\mathbf{H}_{k}^{-1}\right]_{(n, m)} h_{k}^{m, m}\right|^{2} . \\
& b_{k}^{n}=\log _{2}\left(1+\frac{1}{\Gamma\left(\beta_{k}^{\text {diag }}\right)^{2}} \frac{\left|h_{k}^{n, n}\right|^{2} \tilde{s}_{k}^{n}}{\sigma_{k}^{n}}\right) .
\end{aligned}
$$

Tomlinson-Harashima pre-coder: It is non linear pre-coder .because of non linearity complexity of the system is quite high. In this method of precoding, channel is decompose using QR decomposition given in equation (8).

$$
\mathbf{H}_{k}^{\dagger}=\mathbf{Q}_{k} \mathbf{R}_{k},
$$

In the given equation $\mathrm{Q}_{\mathrm{k}}$ is a unitary matrix and $\mathbf{R}_{\mathrm{k}}$ is an upper triangular matrix. Input signal is predistorted by pre-coder matrix $\mathrm{Q}_{\mathrm{k}}$. Output of the system is written as

$$
\mathrm{y}_{k}=\mathbf{H}_{k}\left(\mathrm{Q}_{k}\left(\mathrm{x}_{k}\right)^{\mathrm{int}}\right)+\mathrm{z}_{k}
$$


The benefit of this method is that, if the transmitted signal of user $n=1$ is known, then cross talk induced by that user to other user is also known [16]. With this help, the transmitted signal of second user $(n=2)$ can be pre-distorted recursively. So each can feel crosstalk free transmission. Bit rate of the system is given by following equation (10)

$$
b_{k}^{n}=\log _{2}\left(1+\frac{\left|r_{k}^{n, n}\right|^{2} s_{k}^{n}}{\Gamma \sigma_{k}^{n}}\right) \approx \log _{2}\left(1+\frac{\left|h_{k}^{n, n}\right|^{2} s_{k}^{n}}{\Gamma \sigma_{k}^{n}}\right) .
$$

\section{Simulation and Result}

In this section we present the performance evaluation of system model with and without precoding. Twisted pair wire of 24 gauge and $.5 \mathrm{~mm}$ diameter is used for wire line channel. Modelling of channel is done in Matlab for different frequency and different length of wire. For simulation on Matlab, four wire of different length in Feet are taken in account. AWGN noise and FEXT has been considered for the simulation on Matlab 2015. Random binary sequence was generated using 'randi' function and QAM modulation is used for the transmission of data in channel.

All simulation result is observed for downstream vectored DSL. Binder is having four wire of same kind but different in length. Mixed scenario is taken in to account when all user are at different distance from the central office. MIMO channel of 24 gauge twisted pair of four different length $0.5,1,1.5$ and 2 kfeet are simulated on Matlab. Channel transfer function of twisted pair wire is given in equation (11).

$\mathrm{H}(\mathrm{d}, \mathrm{f})=\mathrm{e}^{-\mathrm{d}(\gamma)}$
$\gamma=\sqrt{(R+j \omega L)(G+j \omega C)}$

here $\mathrm{f}$ is the frequency in $\mathrm{Hz}, \mathrm{d}$ is the length of the cable in

kfeet and $\gamma$ is propagation constant given in equation (12) depend on R,L,C,G component of wire. Channel characteristic is shown in Fig 1.

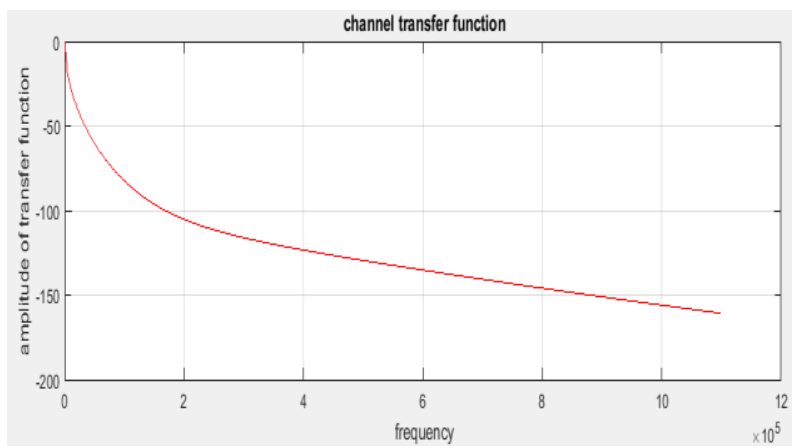

Fig-1.Channel transfer function (24 gauge Twisted pair, 6Kfeet)

The transfer function of channel is decreasing as the frequency of wire is increasing.

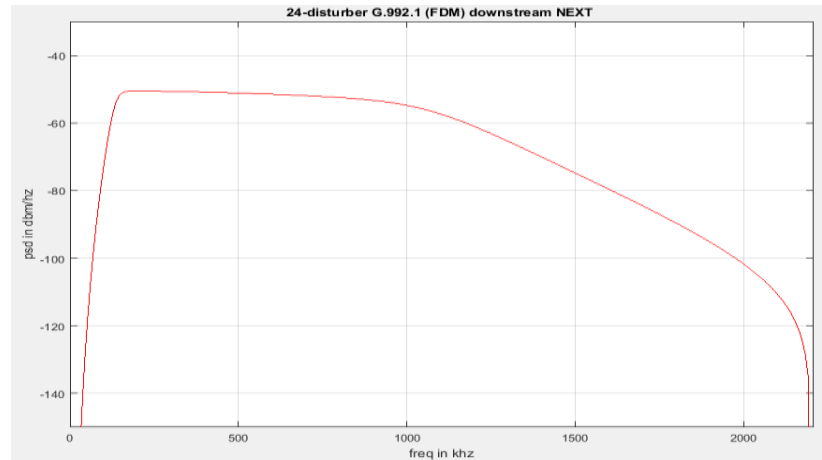

Fig-2. NEXT spectral density of 24 disturber G992.1 Down-stream

Bit rate of vectored DSL with and without precoding is compared. Following observation is noted down. As the length of twisted pair wire is increasing, the bit rate is reducing accordingly. Bit rate without precoding is in the rage of $10^{\wedge} 5 \mathrm{bps}$, there is significant change in bitrate after precoding. 


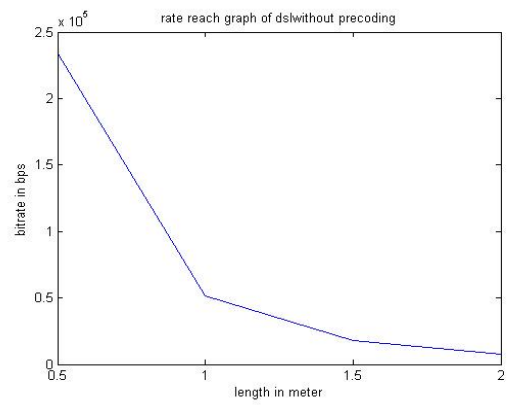

Fig-3.Bit rate for basic model of vectored DSL downstream

The bit rate using zero forcing precoding is achieved in the range of $10^{\wedge} 8 \mathrm{bps}$. Bit rate performance is decreasing as the length of wire increases.

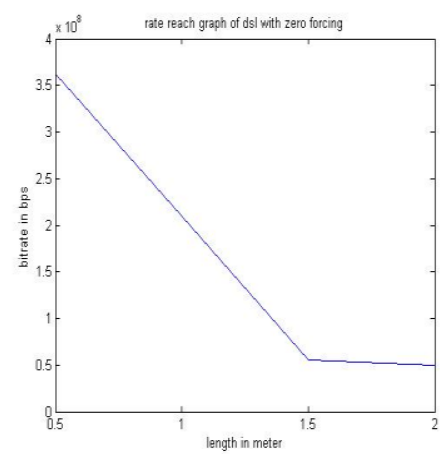

Fig-4.Bit rate using zero forcing pre-coder for vectored DSL downstream

Comparison of bit rate with and without precoding using Matlab.

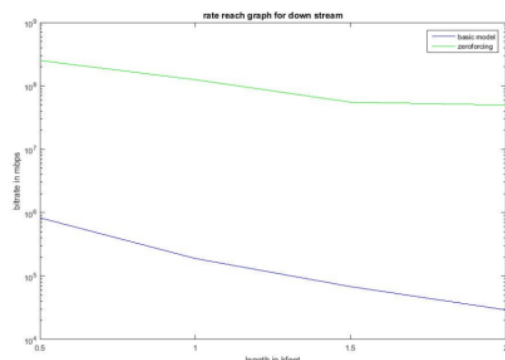

Fig-5.Comparition of bit rate with and without precoding in vecored DSL downstream

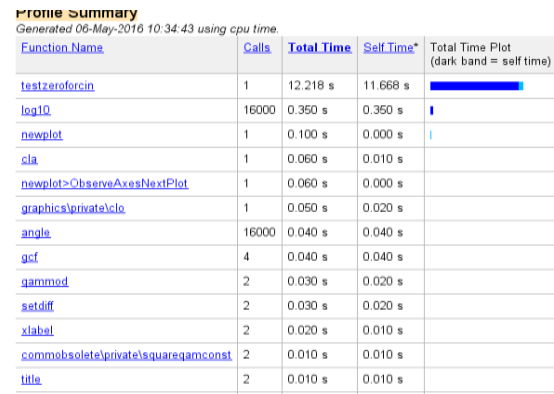

Matlab profiler summary is shown for the comparison of execution time with DSK.

\section{Dsk Simulation Result}

Implementation of basic model of vectored DSL is done using DSK 6713 DSP processor in simulation mode. Complete code is written in c using CCStudio V5.5. The channel is written into a text file "channel. Txt". From txt file in Matlab header file is created include as "channel .h "in c code. Transmission data rate is compared in both Matlab and in DSK platform. Following observation is noted down there is improvement in data rate obtained from DSK then Matlab.as shown in Fig (6). 


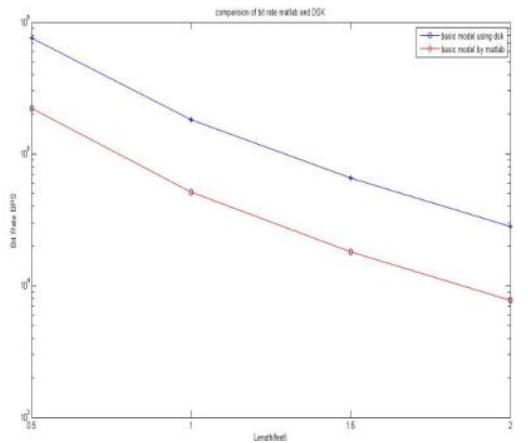

Fig-6.Comparition of bit rate in Matlab and using DSK processor vectored DSL downstream

The computational complexity can be minimized in DSK which is using FFT algorithm. Execution time for DSK is very much less compare to the execution time taken by profiler of Matlab for basic model of vectored DSL.

Table -1: Comparistion Of Execution Time

\begin{tabular}{|l|l|l|l|}
\hline Plateform & \multicolumn{1}{|c|}{$500 \mathrm{KHz}$ Band } & MHz band & $16 \mathrm{MHz}$ band \\
\hline $\begin{array}{l}\text { TMS320C6713 target simulator } \\
\text { basic model }\end{array}$ & $0.306482635 \mathrm{Sec}$ & $0.6049761 \mathrm{Sec}$ & $1.34021 \mathrm{sec}$ \\
\hline Matlab basic model & $1.361 \mathrm{Sec}$ & $2.331 \mathrm{Sec}$ & $4.611 \mathrm{Sec}$ \\
\hline Matlab zero forcing precoding & $1.637 \mathrm{Sec}$ & $12.087 \mathrm{Sec}$ & $12.218 \mathrm{Sec}$ \\
\hline
\end{tabular}

It indicates that implementation in DSK is much faster then matalb.

\section{Conclusion And Future Scope}

Computational complexity is one of the challenges in the design of pre-coder with respect to data rate. In this paper DSK 6713 a digital signal processor is used for implementation of pre-coder in simulation mode. Result shows that there is measurable difference in the execution time using with DSK in compare to Matlab. We can proceed further to implement linear pre-coder on DSP processor (DSK Board). Performance of bit rate and complexity of the vectored DSL system can be analyzed using more number of user in a binder. Observation can be done in scenario when wires in binder can be of different type. Transmission rate can be observed in the presence of non vectored line.

\section{References}

[1] Philip Golden, Herve, Dedieu, Krista Jacobsen “Fundamental of DSL Technology “,Auerbach Publication 2006.

[2] Christopher Leung, Sean Huberman, Khuong Ho-Van, and Tho Le-Ngoc" Vectored DSL:Potential, Issues and Challenges" IEEE Communications surveys \& tutorials, vol. 15, no. 4, fourth quarter 2013.

[3] R. Zidane, S. Huberm C. Leung, and T. Le-Ngoc, "Vectored DSL:Benefits and Challenges for Service Providers," IEEE Commun. Mag, Feb. 2013.

[4] George Ginis and John M. Cioffi " A Multi-user Precoding Scheme achieving Crosstalk Cancellation with,” Application to DSL Systems research gate 2000

[5] R. Cendrillon, F. Liming, J. Chou, G. Long and D. Wei, "Challenges and Solutions in Vectored DSL," in Access , ser. Lecture Notes of the Institute for Computer Sciences, Social Informatics and Telecommunications Engineering. Springer Heidelberg, 2010, vol. 37, pp. 192-203.

[6] R. Cendrillon, G. Ginis, E. Van den Bogaert, and M. Moonen, "A Near-Optimal Linear Crosstalk Pre-coder for Downstream VDSL," IEEE Trans. Commun., vol. 55, no. 5, pp 860-863, May 2007.

[7] S. Huberman, C. Leung, and T. Le- Ngoc, "Dynamic Spectrum Management (DSM) Algorithms for Multi-User xDSL ," IEEE Commun. Surveys Tuts., vol. 14, no. 1, pp. 109-130, Feb. 2012.

[8] I. Bergel and A. Leshem, "The performance of zero forcing DSL systems," IEEE Trans. Signal Process ., vol. PP, 2012.

[9] Fernando P'erez-Cruz, Miguel R. D. Rodrigues, Sergio Verd'u” Optimal Precoding for Digital Subscriber Lines "This full text paper was peer reviewed at the direction of IEEE Communications Society subject matter experts for publication in the ICC 2008 proceedings

[10] R. Cendrillon, G. Ginis, E. Van den Bogaert, and M. Moonen , "A Near- Optimal Linear Crosstalk Canceler for Upstream VDSL," IEEE Trans. Signal Process., vol. 54, no. 8, pp. 3136-3146, Aug. 2006

[11] G. Ginis and J. Cioffi, "Vectored Transmission for Digital Subscriber Line Systems," IEEE J. Sel. Areas Commun vol. 20, no. 5, pp. 1085-1104, Jun. 2002.

[12] ITU-T standard G.test.bis.

[13] ITU-T Recommendation G.993. .1-2004: Very high speed Digital Subscriber Line . SERIES G: Transmission Systems and Media Digital Systems and Networks, 2004.

[14] A. Leshem and L. Youming, "A Low Complexity Linear Precoding Technique for Next Generation VDSL Downstream Transmission Over Copper," IEEE Trans. Signal Process ., vol. 55, no. 11, pp. 5527-5534, Nov. 2007.

[15] H. V. Henderson and S. R. Searle ," On Deriving the Inverse of a Sum of Matrices," SIAM Review, vol. 23, no. 1, pp. 53-60, Jan. 1981.

[16] R. Cendrillon, M. Moonen, T. Bostoen, and G. Ginis The linear Zero-Forcing crosstalk canceller is nearoptimal in DSL channels. In IEEE Global Communications Conference, Dallas, TX, page 5, 2004 